\title{
Outcomes of inpatient psychiatric treatment for adolescents: A multiple perspectives evaluation
}

\begin{abstract}
Adolescent inpatient psychiatric treatment was evaluated from the multiple perspectives of clinicians, young people, and parents using standardised measures and goal-based outcomes (GBOs). The sample included cases $(N=128)$ discharged from a London adolescent unit between April 2009 and December 2015. Measures were completed at admission and discharge, and change in ratings analysed to assess treatment outcomes. Ratings of clinicians and young people on the Health of the Nation Outcome Scales for Children and Adolescents (HoNOSCA) were compared. Adolescents demonstrated significant improvement across all measures from admission to discharge. Correlation between clinicians' and adolescents' HoNOSCA ratings was weak at admission $(r=$ $.25)$ but stronger at discharge $(r=.63)$. Standardised effect sizes were larger for GBOs ( $d=1.73$ and 3.16 for adolescent and clinician-rated goals respectively) compared to all standardised measures $(d=0.31-0.93)$. Improvement was observed across all measures of functioning and symptoms following inpatient treatment. Clinicians and young people developed better shared understanding of the problems from admission to discharge. GBOs are more sensitive to change compared to standardised measures and may be meaningfully adopted by inpatient units for routine outcome monitoring.
\end{abstract}




\section{Keywords}

Adolescent, inpatient, outcomes, HoNOSCA, GBOs

Child and adolescent mental health services (CAMHS) in the United Kingdom are divided into a four-tier structure (Health Advisory Service, 1995). Inpatient services fall under Tier 4 of this framework and provide highly specialised care for young people with the most severe and persistent mental health disorders.

Early reviews (Blotcky, Dimperio, \& Gossett, 1984; Pfeiffer and Strzelecki, 1990) concluded that children and adolescents demonstrated significant improvement following inpatient treatment. However, most studies assessed outcomes only at discharge, with few adopting a pretest-posttest design (Pfeiffer \& Strzelecki, 1990). In addition, few studies utilised standardised measures, making it difficult for comparisons across studies.

Later studies addressed these limitations with more rigorous research designs, as well as standardised measures for assessment of change. Many studies employed clinician ratings on the Children's Global Assessment Scale (CGAS; Shaffer et al., 1983) and the Health of the Nation Outcome Scales for Children and Adolescents (HoNOSCA; Gowers et al., 1999). Jaffa and Scott (1999) found that adolescent inpatients in a Cambridge unit demonstrated significant improvement on clinician-rated CGAS between admission and discharge, as well as at follow-up 6 to18 months later. 
Similarly, Corrigall and Mitchell's (2002) study of 27 admissions to one unit and Tulloch et al.'s (2008) multicentre study across 40 units reported that adolescents achieved significant improvement on both the CGAS and HoNOSCA at discharge compared to admission. Together, these studies provided strong evidence that adolescents' symptoms and functioning tend to improve over the course of inpatient treatment, but were limited in that only clinician-rated outcomes were examined.

Green et al. (2001) and Green et al. (2007) expanded on the above studies by adopting a multiple-perspectives approach and examined outcomes of inpatient treatment from the different viewpoints of clinicians, parents, teachers, and an independent researcher. Both studies highlighted consensus among different informants that children and adolescents demonstrated significant improvement following inpatient treatment.

Using the same sample as Green et al. (2007), Jacobs et al. (2009) employed the Health Needs approach (Kroll, Harrington, \& Bailey, 2000) to assess the impact of inpatient treatment on adolescents' needs and functioning across different psychosocial domains. The study similarly reported that adolescents achieved improvement across different domains from admission to discharge, with further gains observed at followup. Importantly, Jacobs et al. built on previous studies by obtaining outcome ratings from adolescents' perspective in addition to those of clinicians and parents. However, the study synthesised information across different perspectives by using only the highest 
severity ratings across all informants at each time point, which did not differentiate the unique perspective of young people. In line with the growing emphasis within mental health services of taking patients' perspective into account (Hansen et al., 2010; Wolpert et al., 2014), the current study evaluates the outcomes of inpatient treatment separately from the perspectives of young people, clinicians, and parents.

Previous studies were also limited in that different outcome measures were used for different informants, which restricted direct comparisons. In the development of the self-report version of the HoNOSCA (HoNOSCA-SR; Gowers et al., 2002), the authors directly compared self-ratings of adolescents within an inpatient unit to that of clinicians. Correlation between clinician and adolescent self-report ratings was weak at admission $(r=.27)$ but stronger at discharge $(r=.58)$.

On the other hand, a recent study by Yuan (2015) reported weak and nonsignificant correlations between clinicians' and adolescents' HoNOSCA ratings at both admission $(r=.02)$ and discharge $(r=.28)$. However, the $95 \%$ confidence intervals were relatively wide as a result of small sample sizes, $n=74,95 \%$ CI $[-.21, .24]$ at admission; $n=42,95 \%$ CI [-.03, .54] at discharge. This highlights substantial uncertainty associated with the reported point estimates, even before additional factors such as nonresponse rates are taken into consideration. The present study will contribute to this growing area of research by directly comparing clinicians' and adolescents' ratings on the HoNOSCA. 
Beyond standardised measures, there has been increasing interest in recent years towards use of goal-based outcomes (GBOs) for the assessment of treatment outcomes. In contrast to generic outcome measures, GBOs are tailored to the specific difficulties and therapeutic aims of each individual patient; representing a more targeted way of tracking treatment progress (Weisz et al., 2011). Rothery et al. (1995) developed a framework of four major categories of treatment goals and employed it for treatment planning across several adolescent inpatient units. Goals were specified by the clinician for each adolescent at admission and achievement of each goal was rated at discharge. Clinicians in the study rated adolescents to have demonstrated substantial achievements on all goal categories at discharge.

Wolpert et al. (2012) directly compared GBOs with standardised outcome measures for assessment of change following treatment within 41 CAMHS outpatient units. Up to three treatment goals were jointly identified by clinicians, young people, and their family members at the beginning of treatment. Achievement on each goal was rated by clinicians at the start and end of interventions. In line with Rothery et al.'s (1995) study, Wolpert et al. found that young people demonstrated significant progress towards goal achievement following treatment. Importantly, the study established a significant correlation between change in goal ratings and clinician-rated CGAS scores $(r=.38, p<.001)$. The authors argued that this provided support for the construct 
validity of GBOs and justifies their use in conjunction with standardised measures to offer an additional perspective for the evaluation of treatment outcomes.

In contrast, a weaker and nonsignificant correlation $(r=-.16)$ was found between change on GBOs and adolescent self-rated SDQ total severity index (Wolpert et al., 2012). Based on their findings, Wolpert et al. hypothesised that although goals were developed collaboratively by different informants, they might reflect clinicians' aims to a larger extent than those of young people. This runs contrary to Law's (2013) argument that GBOs should ideally be owned by and reflect what adolescents hope to achieve from treatment rather than clinicians. The present study specifically addresses this issue by investigating achievement on goals specified by clinicians and adolescents separately.

\section{Methods}

\section{Study setting}

The study was conducted at Simmons House Adolescent Unit (www.simmonshouse.org) in London. Simmons House is a National Health Service (NHS) inpatient psychiatric unit that caters for 12 young people aged between 13 and 18 years old. 
The unit works with adolescents with a wide range of symptoms, histories, contexts, and needs. These may include symptoms of psychosis, bipolar disorder, obsessive-compulsive disorder, post-traumatic stress disorder, depression, anxieties and/or developmental disorders. The majority of young people come to Simmons House for planned treatment admissions aimed at shifting their developmental trajectory. The unit also accepts emergency admissions for adolescents in acute crisis. Some emergency admissions convert into treatment cases along the way and stay on in the unit for more intensive, long-term therapeutic work. The length of admission varies according to clinical need and context. Young people admitted as emergencies might spend only a few days or weeks on the unit; those admitted for outcome-focused treatment typically have admissions between six to eight months, or longer.

Simmons House is staffed by a multidisciplinary team including psychiatrists, nurses, clinical psychologists, psychotherapists, occupational therapists, and social workers. A wide range of treatments are offered, including psychiatric assessment and medication, individual therapy from different therapeutic models, and family therapy. All treatments are tailored to the specific needs of each adolescent and family. Each young person has a case manager from the multidisciplinary team who coordinates their care and offers a supportive relationship throughout their admission. The unit runs a weekday programme that includes education, group therapies, and daily community 
meetings. Much attention is given to the therapeutic milieu as an essential part of adolescent inpatient care (Kurtz, 2009).

\section{Sample}

The unit has been collecting routine outcome measures since April 2009. Cases discharged between April 2009 and December 2015, with any available outcome data, were included in the current study. Twenty-five cases discharged within this period did not have any outcome data and were therefore excluded. Several adolescents had repeat admissions but each admission was considered as a separate case. The final sample consisted of 128 admission episodes from 113 adolescents.

As in most clinical services, the unit faced real world difficulties collecting outcome data at both admission and discharge. The final sample of 128 cases thus contained a substantial amount of missing data. This was especially common for cases discharged between 2009-2012, during the first three years of routine outcome monitoring. The sample size available thus varied between different analyses.

Ethical approval for this service evaluation was obtained from the Whittington Health Clinical Governance Department. During the admission process, young people and their parents or carers provided informed consent for routine outcome data to be used anonymously for the purposes of audit, service evaluation, and research. 


\section{Measures}

Clinicians, adolescents, and their caregivers completed several standardised measures and GBOs within two weeks of admission and again at discharge.

CGAS (Shaffer et al., 1983). The CGAS is a clinician-rated global assessment of young people's psychosocial functioning. Adolescents' lowest level of functioning within a given time period was rated on a scale of 1 to 100 , which is divided into deciles. Extensive research into its psychometric properties has confirmed that the CGAS has acceptable interrater reliability and is sensitive to change (Rey et al., 1995; Shaffer et al., 1983; Steinhausen, 1987; Weissman, Warner, \& Fendrich, 1990). The consultant psychiatrist in charge of each case rated the CGAS at both admission and discharge. The consultant psychiatrists did not change during the study period and thus CGAS ratings were completed by the same clinician at both time points for all cases.

HoNOSCA (Gowers et al., 1999). The HoNOSCA is a brief clinician-rated scale. Section A consists of 13 items, each capturing a difficulty commonly experienced by children and adolescents within mental health settings. The 13 items can be summed to give a total score of overall severity of problems. There is good evidence that HoNOSCA items have acceptable interrater reliability (Hanssen-Bauer et al., 2007), and that its total score is sensitive to change within both outpatient (Garralda, Yates, \& Higginson, 2000; Gowers et al., 1999) and inpatient settings (Harnett et al., 2005; Urben 
et al., 2015). Each young person's case manager and senior doctor completed the HoNOSCA as a pair on admission and discharge. While the case manager and senior doctor for most cases were the same clinicians at admission and discharge, this was not always possible due to staff changes.

HoNOSCA-SR (Gowers et al., 2002). The HoNOSCA-SR is the adolescent self-report version of the HoNOSCA. The 13 items in the scale parallel those in Section A of the clinician-rated version but were reworded as questions. High test-retest reliability $(r=$ .806) has been reported for the total severity score (Gowers et al., 2002).

Strengths and Difficulties Questionnaire (SDQ; Goodman, 1997; Goodman, Meltzer, \& Bailey, 1998). The SDQ consists of 25 items that assess the strengths and difficulties of children and adolescents, and is divided into five subscales - emotional symptoms, conduct problems, inattention-hyperactivity, peer problems, and prosocial behaviour. Adolescents and their caregivers completed the self and parent versions of the SDQ respectively. In this study, we used the Total Difficulties score, which is obtained as the sum of the first four subscales. In line with Green et al.'s (2007) study, we used raw scores to track changes in outcomes for adolescents with severe difficulties. Total score for each subscale was prorated if at least three out of five of the items were completed (Youthinmind Ltd, 2016). Cases with more than two missing items on any subscale contributing to the Total Difficulties score were excluded from analysis. 
GBOs. The framework proposed by Law (2013) was used. Clinicians and adolescents respectively identified a maximum of three treatment goals within two weeks of admission. Achievement of each goal was rated on a scale of 0 (not at all) to 10 (fully met) at admission and again at discharge. Adolescents' goals were collected throughout the study period; clinicians' goals were introduced and systematically recorded from 2011. Case managers developed clinicians' goals at admission and helped adolescents in the generation of their goals. A typical clinician goal might be: "for the young person to develop strategies to regulate his/her emotions without the need for self-harm”. Typical young person goals might be: "to feel better" or "to be able to spend time with my friends again".

\section{Analysis}

All statistical analyses were conducted using IBM SPSS Statistics for Windows, Version 22.0. To control for inflated Type I error due to multiple tests, a stringent significance level of $p<.01$ (two-tailed) was used and 99\% confidence intervals calculated throughout (cf. Colquhoun, 2014).

Each outcome measure was analysed using only cases with both admission and discharge ratings. As such, the sample size for each outcome measure was different and smaller than the overall sample size. Change on each standardised measure was analysed using separate paired-samples $t$ tests. To compare ratings of different 
respondents, paired-samples $t$ tests as well as Pearson's correlation coefficients were calculated between clinicians' scores on the HoNOSCA and young people's scores on the HoNOSCA-SR at each time point.

With respect to GBOs, average ratings for each case at each time point were obtained by aggregating across the maximum of three goals specified. Change in ratings was calculated by taking the difference between average ratings obtained at discharge and admission. Separate paired-samples $t$ tests were used to investigate change in achievement for goals developed by clinicians and adolescents respectively. Pearson's correlation coefficients between change scores on GBOs and other standardised measures rated by each respective informant were computed to assess correlations between improvements captured by different outcome measures.

Paired-samples $t$ tests may be considered to have two limitations with respect to our data: First, they treat each admission as an independent case, thus ignoring that some admissions refer to the same adolescents. Second, they only analyse cases where both admission and discharge ratings were present, and discard cases that have only one of the two scores. As a sensitivity analysis, we repeated all analyses reported in Tables 2, 5, and 6 using mixed effects models, with a random intercept for the adolescents. Mixed effects models take account of the dependency between observations that arises when the same adolescent is represented in the data set with more than one admission episode. Cases with incomplete data can be included in the analyses. Since results from 
the mixed effects models did not differ substantially from those of the paired-samples $t$ tests, we only reported the latter in this paper. Full results are available from the corresponding author on request.

Where sufficient data were available, we explored the relationships between change in ratings and type of admission and change in ratings and length of stay. We had no hypotheses about these relationships before seeing the data. Therefore, these analyses were exploratory only, and we did not carry out statistical significance tests.

\section{Results}

\section{Sample Characteristics}

The final sample consisted of 128 cases, of which 37 (28.9\%) were male and 91 (71.1\%) female. Age at admission ranged from 12.8 to 18.0 years $(M=16.1, S D=$ $1.33) ; 110(85.9 \%)$ cases were first time admissions to the unit, while $14(10.9 \%)$ and four $(3.1 \%)$ were second and third time admissions respectively. Ninety-three cases were treatment admissions (72.7\%), while the remaining 35 cases were emergency admissions $(27.3 \%)$.

Length of admission for the entire sample ranged from 1 to 609 days $(M=162.12$, $S D=136.48 ;$ the 25 th, 50 th, and 75 th percentiles were $41.50,138.00$, and 252.75 respectively). Admission length for treatment cases spanned the entire range of 1 to 609 
days $(M=208.25, S D=130.31)$, while that for emergency cases ranged from 3 to 281 days $(M=39.54, S D=48.33)$.

Table 1 shows the number and percentages of cases with available data at admission, discharge, and paired across both time points for each outcome measure. The overall sample included a substantial amount of missing data: Paired admission and discharge scores were missing for about a quarter of the 128 cases for both clinicianrated standardised measures, while fewer than half of the cases had paired scores across all adolescent and parent-rated measures. Each subsequent analysis included only cases with available data for the specific measures investigated.

\section{Change on Standardised Outcome Measures from Admission to Discharge}

Significant improvement was observed across all standardised outcome measures from admission to discharge (see Table 2). Estimated effect sizes (Cohen's $d$ ) were 0.93 and 0.90 for clinician-rated CGAS and HoNOSCA respectively, -0.60 for HoNOSCA-SR, 0.31 for self-rated SDQ, and -0.65 for parent-rated SDQ. Figure 1 illustrates the distribution of CGAS change scores. More than half (53.5\%) of the cases had a 10 point or greater increase in CGAS rating that represents a definitive move into a higher descriptive level of functioning.

Exploratory analyses that separate treatment and emergency admissions are presented in Table 3. The data on CGAS show that average functioning is better at 
discharge compared to admission for both treatment and emergency cases, but the mean change is larger for treatment cases. Treatment cases tend to start at lower levels of functioning than emergency cases, but at discharge, the two groups had similar mean levels of functioning. There is not much difference between treatment and emergency cases in mean HoNOSCA ratings, either at admission or discharge. Both groups had improved mean HoNOSCA ratings at discharge, and the amount of change was similar (5.79 and 5.87 points reduction in mean HoNOSCA score for treatment and emergency cases respectively).

Table 4 shows correlations between length of stay at the unit and change in outcome ratings. This demonstrates that longer stay was associated with more improvement in six of the seven outcome measures. The association was strongest for GBOs (both clinician and self-rated) and CGAS ratings. Our data did not indicate that there was an association between length of stay and adolescent-rated SDQ.

\section{Comparisons of Clinicians' and Adolescents' HoNOSCA Ratings}

Table 5 presents mean differences and correlations between clinician and adolescent HoNOSCA ratings. At admission, the mean clinician rating was significantly higher than that of adolescents, mean difference $=6.16,99 \%$ CI $[3.42,8.90]$, with a corresponding effect size of 0.77 . At discharge, the difference between clinicians' and adolescents' ratings was smaller and nonsignificant, mean difference $=2.24,99 \%$ CI [- 
0.50, 4.97]. There was no significant difference in mean HoNOSCA change scores from admission to discharge between the two informants $(p=.075)$.

Correlation between clinician and adolescent ratings at admission was weak and nonsignificant, $r=.25,99 \% \mathrm{CI}[-.02, .48]$. In contrast, significant moderate correlations were found between ratings of the two informants at discharge, $r=.63,99 \%$ CI $[.37$, .80]; as well as change from admission to discharge, $r=.55,99 \%$ CI $[.21, .77]$.

Change on GBOs from Admission to Discharge

Average goal ratings of both clinicians and adolescents (Table 6) demonstrated significant improvement from admission to discharge $(d=3.16$ and 1.73 for clinician and adolescent-rated goals respectively). Effect sizes of change on both clinician and adolescent-rated GBOs were much larger compared to those observed for standardised outcome measures.

Correlations Between Change on GBOs and Standardised Outcome Measures

Correlations between change in average goal ratings and standardised outcome measures rated by the same respective informants are shown in Table 7. Looking at clinician ratings, the evidence points to moderate correlations between change in goal ratings and change scores on both the CGAS, $r=.40,99 \%$ CI $[.07, .65]$; and HoNOSCA, $r=-.37,99 \%$ CI [-.63, -.03]. In contrast, there was weak evidence for a correlation between change on adolescent-rated goals and the HoNOSCA-SR, $r=-.44$, 
99\% CI [-.75, .02]; and no evidence for a correlation between adolescent-rated goals and the self-rated SDQ, $r=-.03,99 \%$ CI $[-.47, .42]$.

\section{Discussion}

This is the first study to consider clinician, adolescent, and parent ratings, and to use goals set and evaluated by adolescents when assessing change in symptoms and functioning following inpatient treatment for adolescents.

\section{Change on Standardised Outcome Measures}

This study has provided strong evidence that mean scores improved substantially from admission to discharge across all standardised outcome measures, including measures of overall functioning and problem severity, and for ratings by clinicians, adolescents, and parents. These findings are in line with those of past reviews (Blotcky et al., 1984; Pfeiffer \& Strzelecki, 1990) as well as more recent studies (Corrigall \& Mitchell 2002; Green et al., 2007; Green et al., 2001; Jacobs et al., 2009; Jaffa \& Scott, 1999; Tulloch et al., 2008).

More than half (53.5\%) of the cases achieved an improvement of 10 points or more on the CGAS between admission and discharge, that has been proposed as the criterion for clinically significant change (Jaffa \& Scott, 1999). As such, it may be 
argued that improvement in functioning of at least $53 \%$ of patients was clinically meaningful according to the CGAS measure.

Within adolescent self-rated measures, the estimated effect size was stronger for the HoNOSCA-SR (-0.60) compared to the SDQ (-0.31). A probable reason might be that the SDQ, by design, does not measure some of the more severe problems experienced within an adolescent inpatient population such as self-harm, disordered eating, substance misuse, or symptoms of psychosis; all of which are explicitly assessed in the HoNOSCA-SR. Nonetheless, substantial change in ratings was found on the parent-rated SDQ $(d=-0.65)$, suggesting that the parent-rated version, at least, is sensitive to change in an adolescent inpatient population.

Exploratory analyses suggested that treatment cases tend to have lower levels of psychological functioning (as measured by CGAS) at admission compared to emergency cases, and that treatment cases tend to improve more. We found no indication of a difference between treatment and emergency cases in terms of psychiatric symptoms (as measured by clinician-rated HoNOSCA) either at admission or discharge. Larger studies are needed to investigate the robustness of these exploratory findings.

Comparisons Between Clinicians' and Adolescents' Perceptions of Difficulties 
At admission, mean clinicians' rating on the HoNOSCA was significantly higher than that of adolescents. Possible explanations might be that young people had a different understanding of, or felt shame about the problems they experienced, and therefore underestimated their level of difficulty. In contrast, difference between mean ratings of the two informants was smaller and nonsignificant at discharge. A reduced mean difference between clinicians' and adolescents' ratings at discharge was also reported by Yuan (2015), although the difference remained substantial and statistically significant in their study.

Furthermore, correlation between ratings of clinicians and adolescents was weak and nonsignificant at admission $(r=.25)$ but stronger and significant at discharge $(r=$ .63). This is in line with Gowers et al.'s (2002) validation study of the HoNOSCA-SR, which reported that correlations between the HoNOSCA and HoNOSCA-SR were .27 and .58 at admission and discharge respectively. These findings might suggest that, for some cases at Simmons House, clinicians and adolescents developed a common understanding and formulation of the problems from admission to discharge. This fits with the clinical aims of the unit's outcome-focused treatment, for young people to have a better understanding of themselves, be better understood by others, and have a more positive developmental trajectory for their future. Achievement of better mutual understanding between clinicians and adolescents may even be argued to represent a positive outcome of inpatient treatment in and of itself. It is known that active 
involvement and "buy-in" of patients enhances therapeutic alliance and is associated with better treatment outcomes (Coulter \& Collins, 2011; Stewart et al., 2000).

\section{Change on GBOs}

In line with progress shown on standardised outcome measures, average goal ratings of both clinicians and adolescents demonstrated significant improvement from admission to discharge. Effect sizes of change for both clinician and adolescent-rated GBOs were much larger than that observed on all standardised outcome measures. This is likely because GBOs were tailored to areas of difficulties and represented specific clinical aims for each individual adolescent. In contrast, standardised outcome measures generally assessed functioning or problems across multiple domains, some of which might not be relevant to a particular adolescent. This supports Weisz et al.'s (2011) proposition that GBOs represent a more focused way of tracking patients' progress compared to standardised measures and underscores the importance and benefits of their use in outcome monitoring.

\section{Correlations Between Change on GBOs and Standardised Outcome Measures}

Statistically significant and moderate correlations were found between change on clinician-rated GBOs and both the CGAS $(r=.40)$ and HoNOSCA $(r=-.37)$. On the other hand, none of the correlations between change in goal ratings and standardised outcome measures completed by adolescents was statistically significant. Nevertheless, 
it is notable that the correlation between change on adolescent-rated GBOs and the HoNOSCA-SR ( $r=-.44$ ) was of a similar magnitude to those observed between GBOs and standardised measures rated by clinicians. As a smaller number of cases contributed to the correlation analysis between adolescent-rated GBOs and the HoNOSCA-SR compared to analyses between clinician-rated measures, the nonsignificant correlation may be due to low statistical power.

A previous study by Wolpert et al. (2012) reported a similar moderate correlation between change in goal ratings and clinician-rated standardised measure $(r=.38)$, but a weaker association between change in goal ratings and standardised outcomes rated by adolescents $(r=-.16)$. Wolpert et al. hypothesised that the jointly agreed goals in their study might have reflected clinicians' aims to a greater extent than those of adolescents. In the present study, separate sets of goals were independently identified and rated by clinicians and young people. Our findings indicate that when goals developed independently by each informant were used, the strength of association between change on adolescent-rated goals and the HoNOSCA-SR was comparable to that between the analogous clinician-rated measures.

An important clinical implication is that adolescents should be given the space, time, and support to identify their goals independently, in order that their goals reflect their own perspective and what they hope to achieve from treatment. Consideration of the differences in change scores between standardised measures such as the HoNOSCA 
and GBOs may also make an important contribution to thinking about what constitutes clinically significant change for adolescents using Tier 4 services. For example, a plateau in symptoms may not be a bad outcome for a young person whose symptoms were deteriorating prior to inpatient admission.

\section{Limitations and Future Directions}

The study was based on routine outcome measures collected at an inpatient unit and employed a single sample pretest-posttest design. No control group or random assignment was conducted given the complex ethical considerations of such a research design with this population. As such, causal conclusions cannot be drawn that inpatient treatment per se led to adolescents' improvement. Change in outcome ratings between admission and discharge may in part reflect regression to the mean or spontaneous recovery.

Clinician measures were rated by clinicians directly involved in adolescents' care as part of routine outcome monitoring. There was thus the possibility of bias as clinicians might unknowingly have been influenced by pressure to demonstrate the effectiveness of treatment provided. This is a limitation of most research using clinician-rated measures.

Adolescents received individualised treatment packages tailored to their specific needs. As such, individual contributions of different treatment components to outcomes 
cannot be separated and the study does not allow for identification of specific intervention or combination of interventions that may have effected adolescents' improvement.

Post discharge follow-up ratings were not available as they were not part of the unit's routine outcome measures collection, which limited assessment of the longerterm effects of inpatient treatment. Future studies should aim to systematically collect follow-up ratings from adolescents, parents, and clinicians to determine the long-term impact of inpatient treatment from multiple perspectives.

Measures of treatment alliance were not included in the unit's routine outcome monitoring. This represents another limitation as alliance measures could have provided corroborative evidence to support the idea that clinicians and young people developed a better shared understanding and formulation of the problems over the course of inpatient treatment. Clinician and adolescent self-report measures of treatment alliance (e.g., Blais, Jacobo, \& Smith, 2010; Haggerty et al., 2015) may be incorporated in the design of future studies. This would also enable investigation of the association between treatment alliance and outcomes of inpatient treatment.

Future studies can also explore the effect of other variables on treatment outcomes. Our exploratory analysis found that longer stay was mildly or moderately associated with more positive change on the CGAS, clinician and self-rated HoNOSCA, 
clinician and self-rated GBOs, and parent-rated SDQ, but not the self-rated SDQ. These findings need corroboration by further studies. Besides length of stay (Green et al., 2007; Jacobs et al., 2009; Pfeiffer \& Strzelecki, 1990), other potential covariates include admission number (first or repeat), level of impairment or symptom severity at admission (Garralda et al., 2000; Jaffa \& Scott, 1999; Yuan, 2015), as well as admission type (treatment or emergency, which we have explored above).

The study was also limited by the amount of missing data. About $25 \%$ of cases did not have paired admission and discharge scores on both clinician-rated standardised measures, while paired scores were available for less than $50 \%$ of cases across all adolescent and parent-rated measures. Adolescents or parents who completed the measures at both time points might have been more engaged with treatment and hence more likely to achieve better outcomes compared to those who did not complete the measures.

\section{Conclusions}

This study provides evidence that young people's average outcome scores improved after treatment in an adolescent inpatient unit, according to the perspectives of clinicians, young people, and parents, and using both standardised measures and ratings of progress towards treatment goals specified separately by clinicians and young people. The correlation between clinicians' and adolescents' HoNOSCA ratings was stronger at 
discharge compared to admission, indicating that they developed a better shared understanding of the problems over the course of inpatient treatment. We observed moderate correlations between change in ratings on GBOs and both the CGAS and HoNOSCA, demonstrating the validity of GBOs for assessing change. Pre-post differences were larger for GBOs compared to standardised measures, likely because GBOs were targeted towards specific difficulties of individual adolescents. These findings underscore the advantage of GBOs and support their inclusion alongside standardised measures for outcome monitoring within Tier 4 inpatient settings. 


\section{Acknowledgements}

The authors would like to acknowledge all patients, families, carers, and clinicians at Simmons House who participated in the collection of routine outcomes.

\section{Funding}

No external funding was obtained for this study.

\section{Declaration of conflicting interests}

(Author declaration withheld for peer review) 


\section{References}

Blais, M. A., Jacobo, M. C., \& Smith, S. R. (2010), Exploring therapeutic alliance in brief inpatient psychotherapy: A preliminary study. Clinical Psychology \& Psychotherapy, 17(5), 386-394.

Blotcky, M., J., Dimperio, T. L., \& Gossett, J. T. (1984). Follow-up of children treated in psychiatric hospitals: A review of studies. The American Journal of Psychiatry, 141(12), 1499-1507.

Colquhoun, D. (2014). An investigation of the false discovery rate and the misinterpretation of p-values. Royal Society Open Science, 1(3), 140216.

Corrigall, R., \& Mitchell, A. (2002). Service innovations: Rethinking in-patient provision for adolescents. The Psychiatric Bulletin, 26, 388-392.

Coulter, A., \& Collins, A. (2011). Making shared decision-making a reality: No decision about me, without me. London, United Kingdom: The King's Fund.

Garralda, M. E., Yates, P., \& Higginson, I. (2000). Child and adolescent mental health service use: HoNOSCA as an outcome measure. The British Journal of Psychiatry, 177, 52-58.

Goodman, R. (1997). The Strengths and Difficulties Questionnaire: A research note. The Journal of Child Psychology and Psychiatry, 38(5), 581-586. 
Goodman, R., Meltzer, H., \& Bailey, V. (1998). The Strengths and Difficulties Questionnaire: A pilot study on the validity of the self-report version. European Child \& Adolescent Psychiatry, 7(3), 125-130.

Gowers, S. G., Harrington, R. C., Whitton, A., Lelliott, P., Beevor, A., Wing, J., \& Jezzard, R. (1999). Brief scale for measuring the outcomes of emotional and behavioural disorders in children. Health of the Nation Outcome Scales for Children and Adolescents (HoNOSCA). The British Journal of Psychiatry, 174, 413-416.

Gowers, S., Levine, W., Bailey-Rogers, S., Shore, A., \& Burhouse, E. (2002). Use of a routine, self-report outcome measure (HoNOSCA-SR) in two adolescent mental health services. The British Journal of Psychiatry, 180, 266-269.

Green, J., Jacobs, B., Beecham, J., Dunn, Graham, Kroll, L., Tobias, C., \& Briskman, J. (2007). Inpatient treatment in child and adolescent psychiatry-A prospective study of health gain and costs. Journal of Child Psychology and Psychiatry, 48(12), 1259-1267.

Green, J., Kroll, L., Imrie, D., Frances, F., M., Begum, K., Harrison, L., \& Anson, R. (2001). Health gain and outcome predictors during inpatient and related day treatment in child and adolescent psychiatry. Journal of the American Academy of Child \& Adolescent Psychiatry, 40(3), 325-332. 
Haggerty, G., Siefert, C. J., Sinclair, S. J., Zodan, J., Babalola, R., \& Blais, M. A. (2015). Validation of a measure of alliance for an adolescent inpatient setting. Clinical Psychology \& Psychotherapy, 22(4), 357-363.

Hansen, L. K., Vincent, S., Harris, S., David, E., Surafudheen, S., \& Kingdon, D. (2010). A patient satisfaction rating scale for psychiatric service users. The Psychiatric Bulletin, 34, 485-488.

Hanssen-Bauer, K., Aalen, O. O., Rudd, T., \& Heyerdahl, S. (2007). Inter-rater reliability of clinician-rated outcome measures in child and adolescent mental health services. Administration and Policy in Mental Health and Mental Health Services Research, 34(6), 504-512.

Harnett, P. H., Loxton, N. J., Sadler, T., Hides, L., \& Baldwin, A. (2005). The Health of the Nation Outcome Scales for Children and Adolescents in an adolescent inpatient sample. Australian and New Zealand Journal of Psychiatry, 39(3), 129135.

Health Advisory Service (1995). Together we stand: The commissioning, role and management of child and adolescent mental health services. London: Her Majesty's Stationery Office. 
Jacobs, B., Green, J., Kroll, L., Tobias, C., Dunn, G., \& Briskmaan, J. (2009). The effect of inpatient care on measured Health Needs in children and adolescents. The Journal of Child Psychology and Psychiatry, 50(10), 1273-1281.

Jaffa, T., \& Scott, C. (1999). Do inpatients on adolescent units recover? A study of outcome and acceptability of treatment. European Child \& Adolescent Psychiatry, 8(4), 292-300.

Kroll, L., Harrington, R., \& Bailey, S. (2000). Needs assessment of children and adolescents. Child Psychology \& Psychiatry Review, 5(2), 81-88.

Kurtz, Z. (2009). The evidence base to guide development of Tier 4 CAMHS. London, United Kingdom: Department of Health.

Law, D. (2013). Goals and goal based outcomes (GBOs): Some useful information. London: CAMHS Press. Retrieved from http://www.corc.uk.net/resources/additional-information-about-themeasures/practitioner/

Pfeiffer, S. I., \& Strzelecki, S. C. (1990). Inpatient psychiatric treatment of children and adolescents: A review of outcome studies. Journal of the American Academy of Child \& Adolescent Psychiatry, 29(6), 847-853. 
Rey, J. M., Starling, J., Wever, C., Dossetor, D. R., \& Plapp, J., M. (1995). Inter-rater reliability of global assessment of functioning in clinical setting. Journal of Child Psychology and Psychiatry, 36(5), 787-792.

Rothery, D., Wrate, R., McCabe, R., Aspin, J., \& Bryce, G. (1995). Treatment goalplanning: Outcome findings of a British prospective multi-centre study of adolescent inpatient units. European Child \& Adolescent Psychiatry, 4(3), 209221.

Shaffer, D., Gould, M. S., Brasic, J., Ambrosini, P., Fisher, P., Bird, H., \& Aluwahlia, S. (1983). A Children's Global Assessment Scale (CGAS). Archives of General Psychiatry, 40(11), 1228-1231.

Steinhausen, H. C. (1987). Global assessment of child psychopathology. Journal of the American Academy of Child \& Adolescent Psychiatry, 26(2), 203-206.

Stewart, M., Brown, J. B., Donner, A., McWhinney, I. R., Oates, J., Weston, W. W., \& Jordan, J. (2000). The impact of patient-centered care on outcomes. The Journal of Family Practice, 49(9), 796-804.

Tulloch, S., Lelliott, P., Bannister, D., Andiappan, M., O’Herlihy, A., Beecham, J., \& Ayton, A. (2008). The costs, outcomes and satisfaction for inpatient child and adolescent psychiatric services (COSI-CAPS) study. London: Her Majesty’s 
Stationery Office. Retrieved from

http://www.rcpsych.ac.uk/pdf/COSI\%20CAPS.pdf

Urben, S., Pihet, S., Graap, C., Baier, V., Dyson, C., Courosse, S., \& Holzer, L. (2015). Clinical utility of the 2 new scales of the Health of the Nation Outcome Scales for Children and Adolescents (HoNOSCA): A naturalistic, prospective study in a psychiatric unit for adolescents. Journal of Psychiatric Practice, 21(3), 232-240.

Weissman, M. M., Warner, V., \& Fendrich, M. (1990). Applying impairment criteria to children's psychiatric diagnosis. Journal of the American Academy of Child \& Adolescent Psychiatry, 29(5), 789-795.

Weisz, J. R., Chorpita, B. F., Frye, A., Ng, M. Y., Lau, N., Bearman, S. K., . . . Hoagwood, K. E. (2011). Youth top problems: Using idiographic, consumerguided assessment to identify treatment needs and to track change during psychotherapy. Journal of Consulting and Clinical Psychology, 79(3), 369-380.

Wolpert, M., Deighton, J., De Francesco, D., Martin, P., Fonagy, P., \& Ford, T. (2014). From 'reckless' to 'mindful' in the use of outcome data to inform service-level performance management: Perspectives from child mental health. BMJ Quality \& Safety, 23(4), 272-276. 
Wolpert, M., Ford, T., Trustam, E., Law, D., Deighton, J., Flannery, H., \& Fugard, R. J. B. (2012). Patient-reported outcomes in child and adolescent mental health services (CAMHS): Use of idiographic and standardized measures. Journal of Mental Health, 11(2), 165-173.

Youthinmind Ltd. (2016). Scoring the Strengths \& Difficulties Questionnaire for age 417 or $18+$. Retrieved from http://www.sdqinfo.org/py/sdqinfo/b3.py?language=Englishqz(UK)

Yuan, J. M. (2015). HoNOSCA in an adolescent psychiatric inpatient unit: An exploration of outcome measures. Psychiatria Danubina, 27(Suppl. 1), 357-363. 


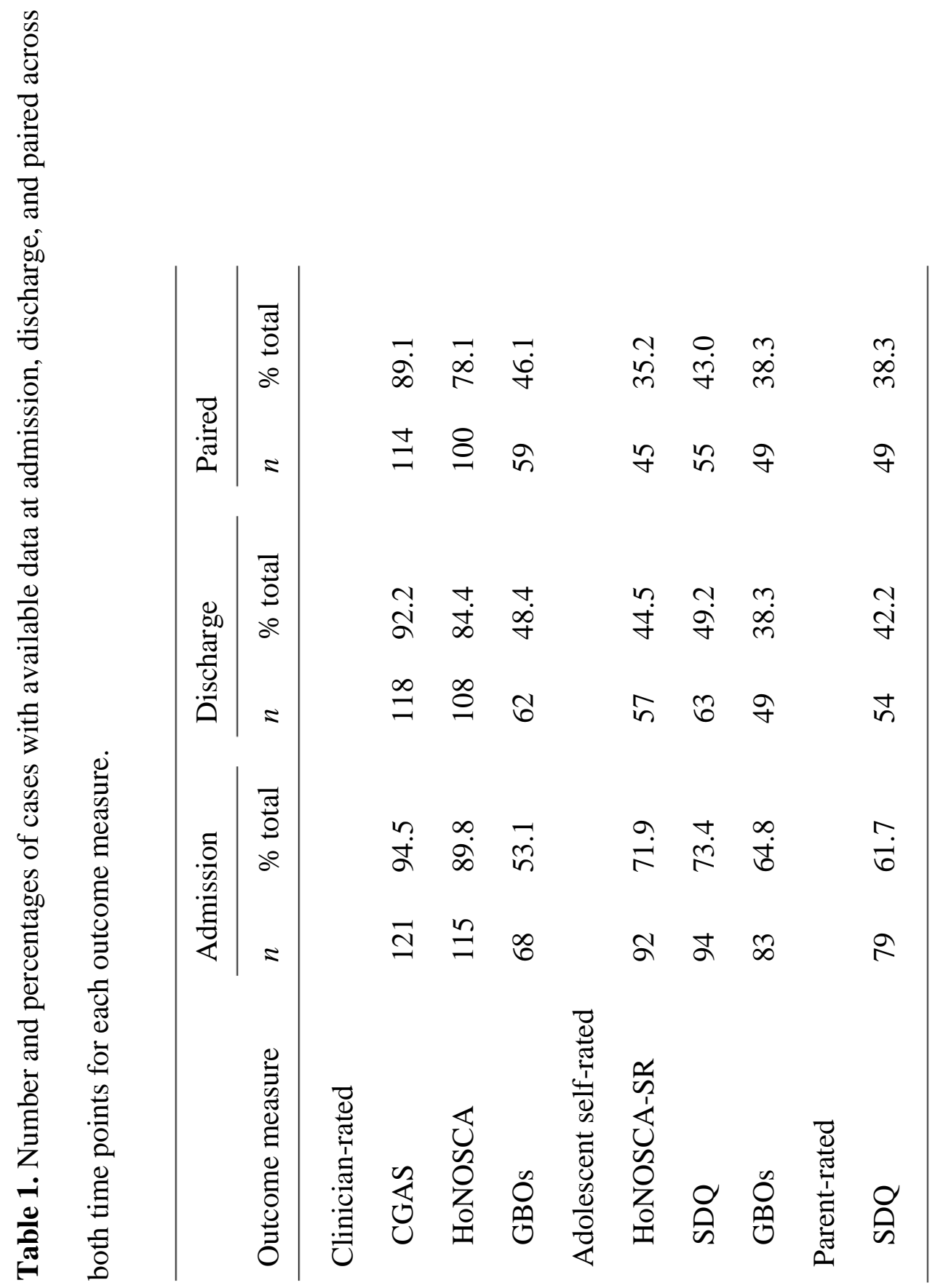




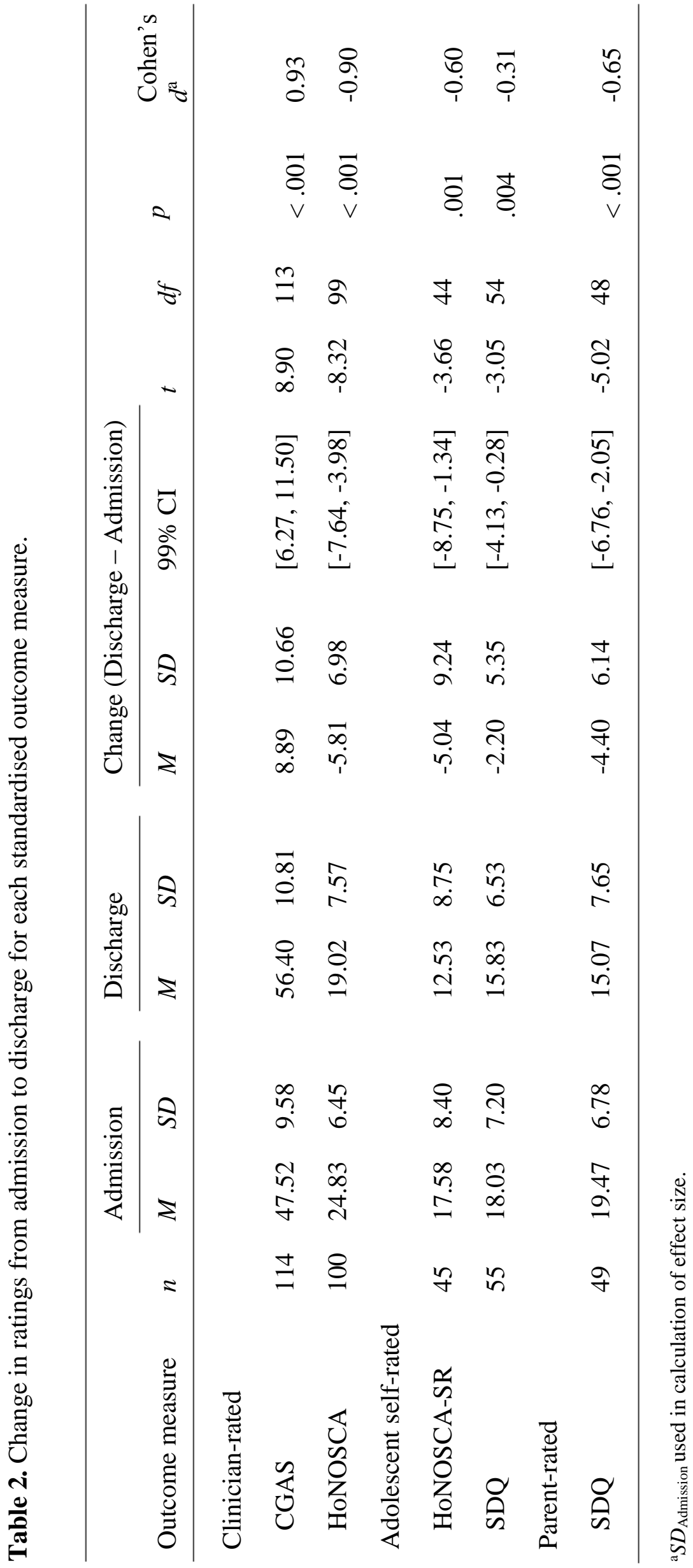


Table 3. Means and standard deviations of CGAS and clinician-rated HoNOSCA for treatment and emergency cases.

\begin{tabular}{|c|c|c|c|c|c|c|c|}
\hline \multirow[b]{2}{*}{ Outcome measure } & \multirow[b]{2}{*}{$n$} & \multicolumn{2}{|c|}{ Admission } & \multicolumn{2}{|c|}{ Discharge } & \multicolumn{2}{|c|}{$\begin{array}{l}\text { Change (Discharge- } \\
\text { Admission) }\end{array}$} \\
\hline & & $M$ & $S D$ & $M$ & $S D$ & $M$ & $S D$ \\
\hline \multicolumn{8}{|l|}{ CGAS } \\
\hline Treatment & 84 & 45.88 & 9.13 & 56.17 & 10.30 & 10.29 & 9.89 \\
\hline Emergency & 30 & 52.10 & 9.46 & 57.07 & 12.29 & 4.97 & 11.89 \\
\hline \multicolumn{8}{|l|}{ HoNOSCA } \\
\hline Treatment & 77 & 24.75 & 6.43 & 18.96 & 7.30 & -5.79 & 7.02 \\
\hline Emergency & 23 & 25.09 & 6.68 & 19.22 & 8.68 & -5.87 & 7.01 \\
\hline
\end{tabular}


Table 4. Correlations between length of stay and change on each outcome measure.

\begin{tabular}{lll}
\hline Outcome measure & $n$ & $r$ \\
\hline Clinician-rated & & \\
CGAS & 114 & .394 \\
HoNOSCA & 100 & -.211 \\
GBOs & 59 & .460 \\
Adolescent self-rated & & \\
HoNOSCA-SR & 45 & -.259 \\
SDQ & 55 & .055 \\
GBOs & 49 & .394 \\
Parent-rated & & \\
SDQ & 49 & -.197 \\
\hline
\end{tabular}




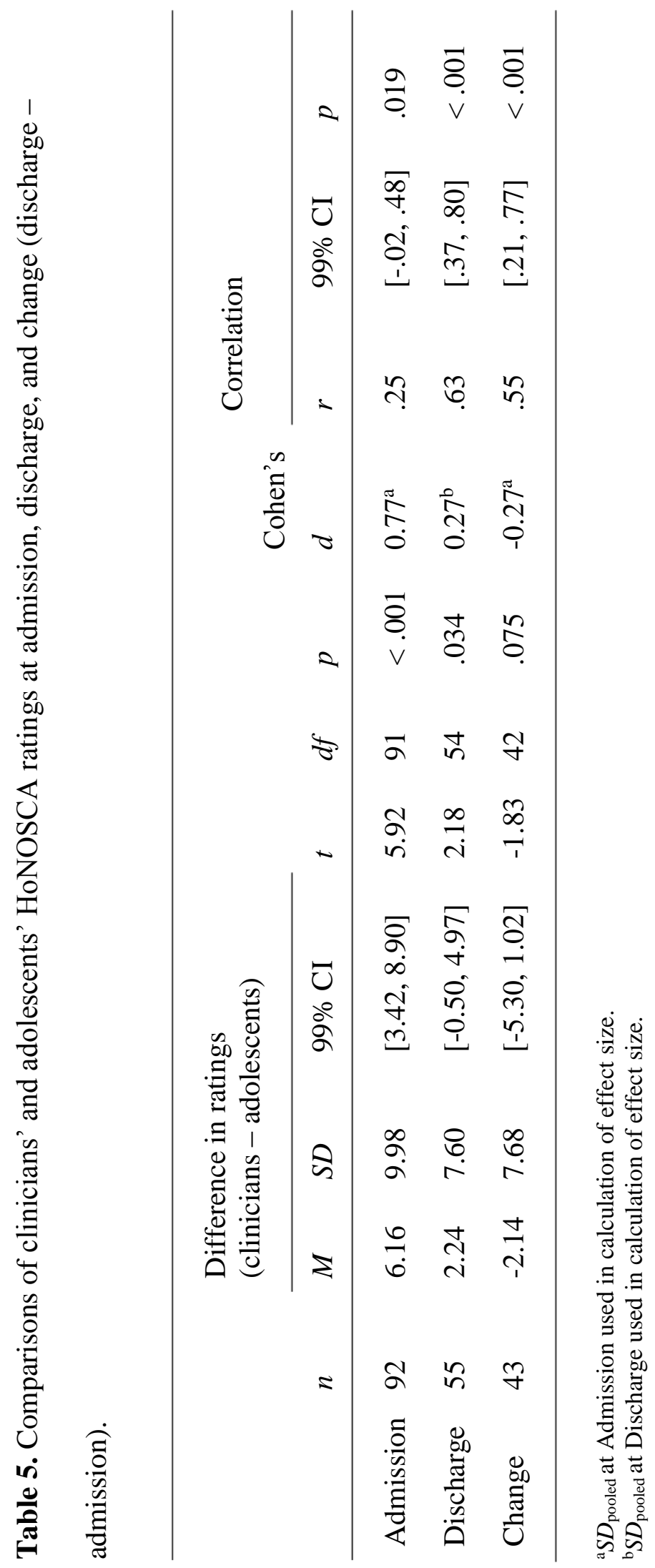




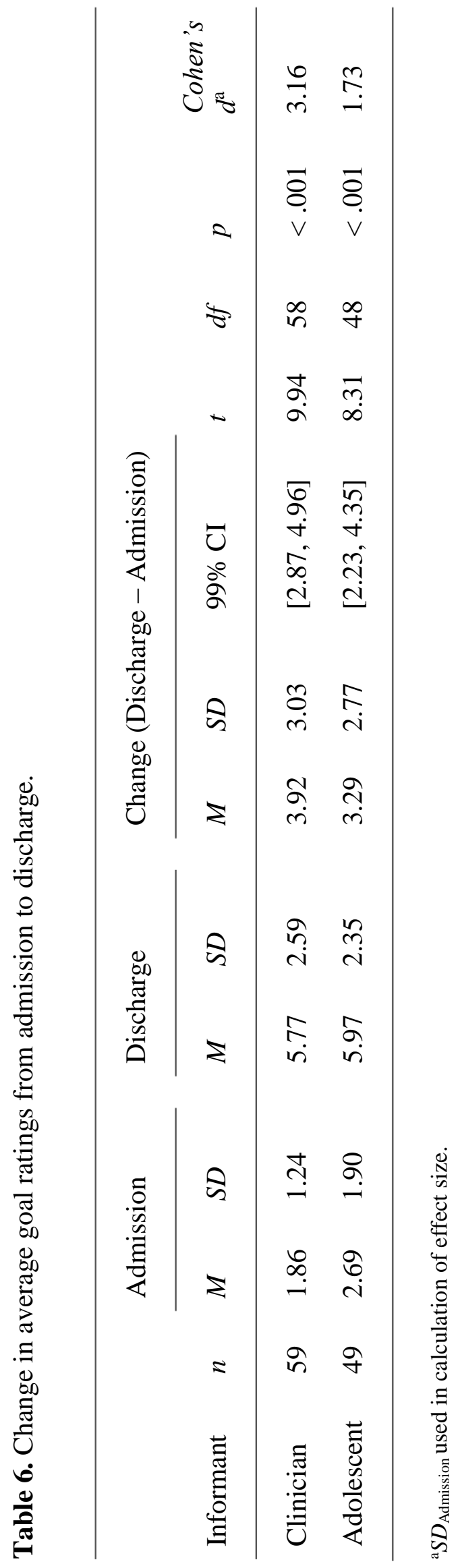


Table 7. Correlations between change on GBOs and standardised outcome measures.

\begin{tabular}{lllll}
\hline & \multicolumn{4}{l}{ Correlation } \\
\cline { 3 - 5 } & $n$ & $r$ & $99 \%$ CI & $p$ \\
\hline Clinician-rated & & & & \\
GBOs and CGAS & 57 & .40 & {$[.07, .65]$} & .002 \\
GBOs and HoNOSCA & 55 & -.37 & {$[-.63,-.03]$} & .006 \\
Adolescent self-rated & & & & \\
GBOs and HoNOSCA-SR & 30 & -.44 & {$[-.75, .02]$} & .015 \\
GBOs and SDQ & 32 & -.03 & {$[-.47, .42]$} & .871 \\
\hline
\end{tabular}




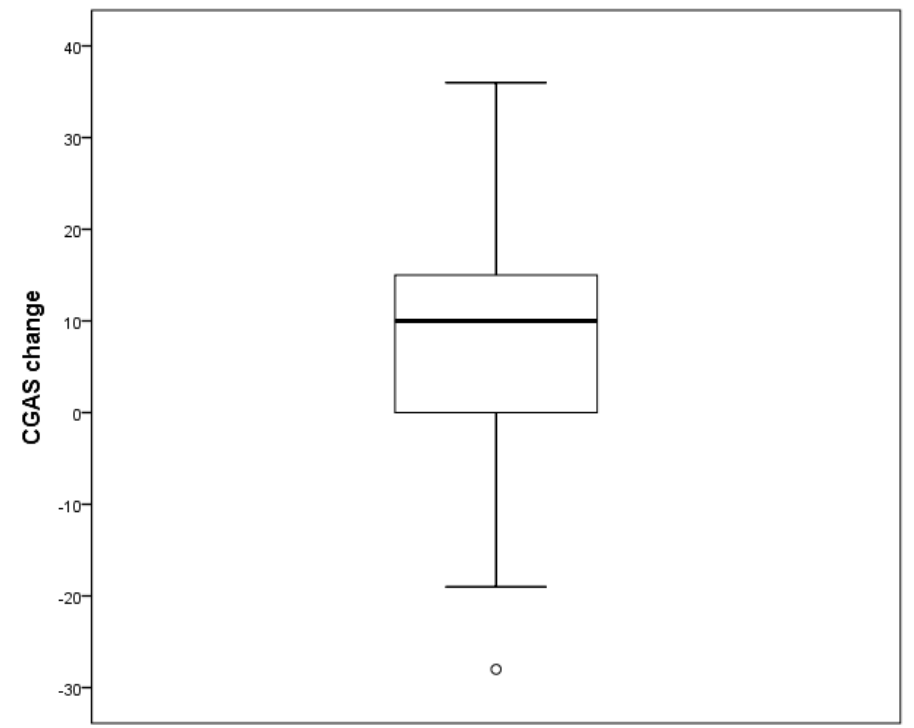

Figure 1. Boxplot of CGAS change scores $(n=114)$. 